\title{
Mechanochemical Activation and Patterning of an Adhesive Surface toward Nanoparticle Deposition
}

\author{
H. Tarik Baytekin, ${ }^{\dagger, \ddagger}$ Bilge Baytekin, ${ }^{\dagger, \ddagger, \S}$ Sabil Huda, ${ }^{\dagger}$ Zelal Yavuz, ${ }^{\ddagger}$ and Bartosz A. Grzybowski*,
}

${ }^{\dagger}$ Department of Chemistry and Department of Chemical and Biological Engineering, Northwestern University 2145 Sheridan Road, Evanston, Illinois 60208, United States

${ }^{\ddagger}$ UNAM-Institute of Materials Science and Nanotechnology, Bilkent University, 06800 Ankara, Turkey

${ }^{\S}$ Department of Chemistry, Bilkent University, 06800 Ankara, Turkey

\section{Supporting Information}

ABSTRACT: Mechanical pulling of adhesive tape creates radicals on the tape's surface. These radicals are capable of reducing metal salts to the corresponding metal nanoparticles. In this way, the mechanically activated tape can be decorated with various types of nanoparticles, including $\mathrm{Au}, \mathrm{Ag}, \mathrm{Pd}$, or $\mathrm{Cu}$. While retaining their mechanical properties and remaining "sticky," the tapes can exhibit new properties derived from the presence of metal nanoparticles (e.g., bacteriostaticity, increased electrical conductivity). They can also be patterned with nanoparticles only at selective locations of mechanical activation.

$\mathrm{D}$ eposition of nanoparticles (NPs) on surfaces is important in a range of technologies, leading to the formation of antibacterial films on clothes and kitchen appliances, ${ }^{1}$ medical devices, ${ }^{2}$ electronic components, ${ }^{3}$ and many more. Other than physisorption, the formation of such NP coatings requires chemical activation of the target surface, enabling the formation of covalent bonds harboring the NPs. Here we take a conceptually different route to surface activation and NP deposition, namely, via mechanochemical treatment. We have recently shown that when contacted and then separated, polymeric surfaces develop both surface charges and radicals. ${ }^{4}$ These species form as a result of bond breaking-heterolytic and homolytic, respectively-that occurs when the materials are being separated. In the present work, we applied these phenomena to popular adhesive tapes, which, when pulled, develop enough mechanoradicals to drive radicalic reduction of metal salts to their nanoparticulate forms. By this route, we were able to deposit a range of different types of NPs-from antibacterial silver ${ }^{6}$ to antifungal copper ${ }^{7}$ on the "sticky" side of the tape or only on its patterned fragments. The tapes covered with NPs retain their adhesive properties while gaining new ones, including increased electrical conductivity or bacteriostaticity. The mechanochemical activation by simple pulling provides a straightforward yet previously unexplored means of surface activation of polymer-based adhesives.

Our material of choice was a commercial Scotch adhesive tape (Figure 1a), the same as the one underlying the Nobelwinning work on graphene ${ }^{8}$ and several other applications such as triboluminescence, nondamaging isolation of plant tissues, and quantification of material adhesion. ${ }^{9}$ Although we used the

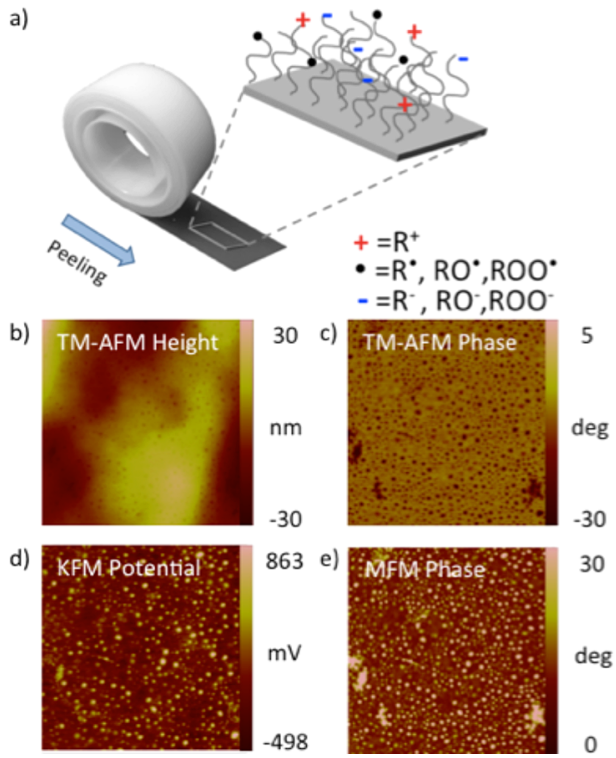

Figure 1. (a) Formation of charged species as well as mechanoradicals on the sticky side of the adhesive tape upon peeling. (b) AFM height image, (c) AFM phase image, and (d) KFM map illustrating the charge mosaics comprising both positive and negative regions. (e) MFM map evidencing the presence of mechanoradicals (white spots). For images of the tape's nonsticky side, see Figure S3. All of the images are $10 \mu \mathrm{m} \times 10 \mu \mathrm{m}$. For chemical tests further confirming the presence of mechanoradicals, see Figure $4 \mathrm{~b}, \mathrm{c}$.

Scotch tape in most of the experiments described below, we also verified the formation of NPs on tapes of different compositions and from different suppliers (e.g., VIBAC 2 " clear packaging tape).

From the standpoint of mechanochemistry, Scotch tape is convenient because the polyacrylic adhesive it contains is in conformal contact with the tape's nonsticky polyethylene-based side. When peeled off with typical speeds measured in $\mathrm{cm} / \mathrm{s}$, the separation of the adhesive results in heterolytic bond breaking and contact charging characterized by a net/ macroscopic charge on the order of few $\mathrm{nC} / \mathrm{cm}^{2}$, as measured using an in-house-made Faraday cage connected to an electrometer. At the microscale, Kelvin force microscopy

Received: August 22, 2014

Published: January 18, 2015 
(KFM), a technique to map surface potentials (for details, see refs $4 \mathrm{~b}$ and $4 \mathrm{~d}$ ), provides evidence that the charging is nonuniform, in the form of charge "mosaics" (Figure 1d). In addition, adhesive/tape separation entails homolytic bond cleavage to yield surface radicals that can be visualized by magnetic force microscopy (MFM), which measures long-range magnetostatic coupling between a magnetized probe/tip and the sample (Figure 1e). ${ }^{5}$ As we have demonstrated before ${ }^{4 a, c}$ for contact charged polymers, it is these mechanoradicals rather than the charges that can drive chemical transformations at mechanically activated polymer-water interfaces; the results below build on these early findings.

In a typical experiment, Scotch tape was manually peeled off (at $\sim 1 \mathrm{~cm} / \mathrm{s}$ ) and then immersed in a $2 \mathrm{mg} / \mathrm{mL}$ aqueous solution of a desired metal salt (here, $\mathrm{AgNO}_{3}, \mathrm{HAuCl}_{4}, \mathrm{PdCl}_{2}$, or $\left.\mathrm{Cu}(\mathrm{acac})_{2}\right)$ for several hours to days. During this time, the tape gradually developed a deep color (pink-red in Au, yelloworange in $\mathrm{Ag}$, gray-brown in $\mathrm{Pd}$, and greenish in $\mathrm{Cu}$ salt solutions; Figure 2a). These colors reflected the formation of

a)

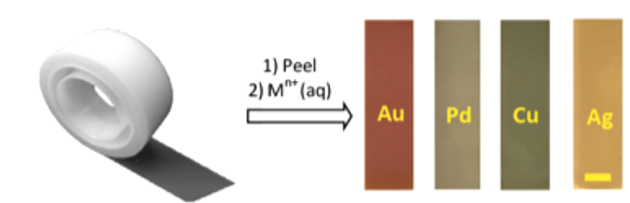

b)
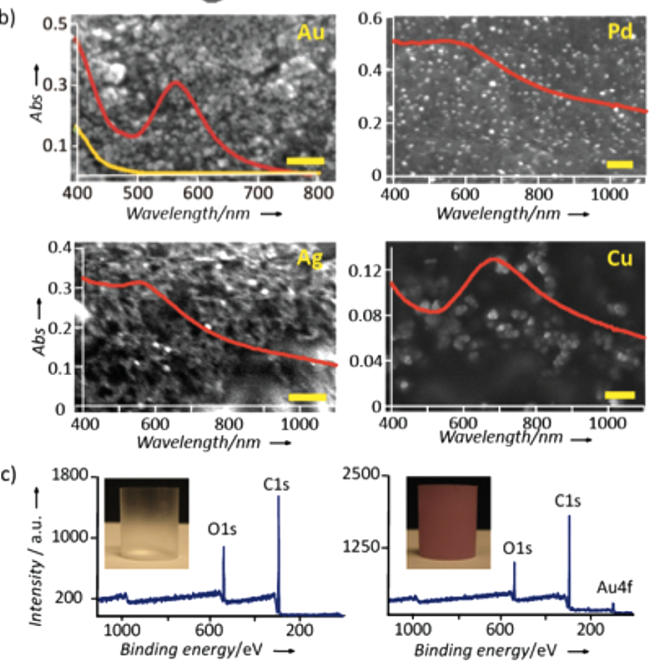

Figure 2. (a) Upon peeling off the tape and immersing it in an aqueous solution of $\mathrm{HAuCl}_{4}, \mathrm{PdCl}_{2}, \mathrm{Cu}(\mathrm{acac})_{2}$, or $\mathrm{AgNO}_{3}$ (each 2 $\mathrm{mg} / \mathrm{mL}$ in $\mathrm{H}_{2} \mathrm{O}$ ), metal NPs were formed on the adhesive sides of the tape. Scale bar $=1 \mathrm{~cm}$. (b) Red curves are the UV-vis absorption spectra of the tapes after 2 days of immersion in different metal salt solutions. The spectra feature characteristic SPR bands, which are absent in the spectra of the salts (e.g., the yellow curve for $\mathrm{HAuCl}_{4}$ in the upper-left picture). The relatively broad SPR bands are expected of both large (ca. $50 \mathrm{~nm}$ ) and aggregated NPs. All types of metal NPs formed on the tapes were imaged by SEM ( $\mathrm{Au}$, top left; Pd, top right; $\mathrm{Cu}$, bottom right; $\mathrm{Ag}$, bottom left). Scale bar $=200 \mathrm{~nm}$, immersion time $=10 \mathrm{~h}$. (c) XPS spectrum featuring the $\mathrm{Au} 4 \mathrm{f}$ peak at $84 \mathrm{eV}$ characteristic of $\mathrm{Au}$ NPs.

metal nanoparticles. The presence of $\sim 100 \mathrm{~nm} \mathrm{NPs}$ was directly confirmed by scanning electron microscopy (SEM), as shown by the images in Figure $2 \mathrm{~b}$ for (clockwise) $\mathrm{Au}, \mathrm{Pd}, \mathrm{Cu}$, and Ag. (For TEM images of NPs see Figure S8). In addition, UV-vis spectra of Au-, Ag-, Pd-, and Cu-covered tapes featured characteristic surface plasmon resonance (SPR) bands indicative of the presence of fully reduced metal NPs; this was also confirmed by X-ray photoelectron spectroscopy (XPS), as shown in Figure $2 \mathrm{c}$ and Figure S10.

The formation of NPs was not accompanied by any significant changes in mechanical properties, which we quantified at the nanoscale by the Derjaguin-Muller-Toporov (DMT) method (this technique determines the reduced modulus, which is roughly comparable to a material's Young's elastic modulus; Figure 3a) and adhesion mapping (Figure 3b)
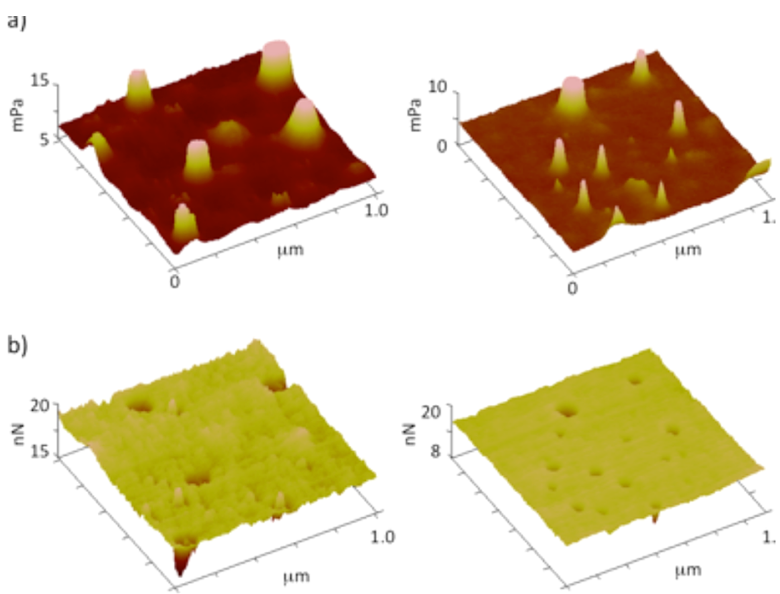

Figure 3. Comparison of tape properties before (left column) and after (right column) formation of nanoparticles as studied by AFM/ PeakForce QNM: (a) DMT modulus; (b) adhesion maps. The similar vertical scales in each pair of images demonstrate that the mechanical properties of the tape surface do not change significantly upon NP formation.

via the PeakForce Quantitative Nanomechanical Measurement (QNM) method. ${ }^{10}$ On the macroscopic scale, after drying, the tapes decorated with NPs remained adhesive to various types of surfaces (Figure S11).

A plausible mechanism by which the metal NPs form on a mechanoactivated tape involves the homolytic cleavage ${ }^{4 a, 11}$ of bonds during peel-off (Figure 4a). It is known that such radicals
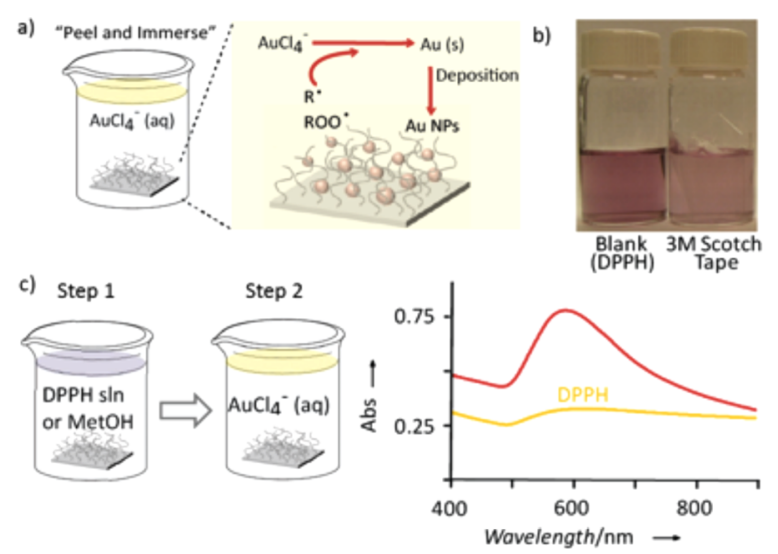

Figure 4. (a) Radicalic mechanism by which nanoparticles form on peeled-off adhesive tapes. (b) Optical images of the DPPH solution (4 $\mathrm{mg} / \mathrm{L}, \mathrm{CH}_{3} \mathrm{CN}$ ) before and after tape immersion for $24 \mathrm{~h}$. (c) The yellow line is a visible spectrum of a peeled-off tape first immersed into a $4 \mathrm{mg} / \mathrm{L}$ DPPH solution for $2 \mathrm{~h}$ and then kept in $2 \mathrm{mg} / \mathrm{mL}$ $\mathrm{HAuCl}_{4}$ (aq) for $24 \mathrm{~h}$. The drastic reduction in the intensity of the $\mathrm{Au}$ SPR peak compared with that for the tape that was not immersed in radical scavenging DPPH (red line) should be noted. 
can reduce metal ions to solid metals, ${ }^{4 a, c, 12}$ which is also facilitated by small amounts of $\mathrm{H}_{2} \mathrm{O}_{2}$ formed in aqueous solutions of mechanically treated polymers. ${ }^{4 a, 13}$ As shown in Figure 1e, mechanoradicals on the peeled-off tape can be detected by MFM. The existence of radicals was also independently confirmed by a chemical test in which the tape was immersed in a solution of the radical scavenger 2,2diphenyl-1-picrylhydrazyl (DPPH) $\left(4 \mathrm{mg} / \mathrm{L}\right.$ in $\left.\mathrm{CH}_{3} \mathrm{CN}\right)$ immediately after it was peeled off; ${ }^{14}$ upon such immersion, the absorption maximum of the solution at $\lambda_{\max }=517 \mathrm{~nm}$ decreases in intensity and the color fades (Figure $4 \mathrm{~b}$ ), indicating that the radical scavenger reacted with the mechanoradicals. The key role of mechanoradicals was also corroborated by experiments in which exposure to DPPH eliminated the ability of the tapes to cause NP formation upon immersion in a salt solution (Figure 4c, red line vs yellow line).

To eliminate the possibility that the acrylate polymer base itself contained the reductant, we removed this polymer from the tape by soaking it in toluene or dichloromethane (see Figures S1 and S2 in the Supporting Information). These solvents did not dissolve the polyethylene backing strip, and the polyacrylate was selectively removed, dried, and redispersed in dichloromethane. When this "recovered" polyacrylate was then treated with various metal salts, no reduction and no formation of metal nanoparticles were observed even after several weeks, emphasizing again the importance of the polymer's mechanical activation.

Finally, we note that we previously confirmed that reduction of metals in an aqueous environment could not be attributed to the charged species on mechanically treated polymers. For details, see refs $4 \mathrm{a}$ and $4 \mathrm{c}$.

We conclude with several considerations of potential practical usefulness. First, the concentration of the formed NPs increases with the immersion time in the salt solution (Figure 5a and Figure S6). Second, the tapes can be patterned with NPs only in select locations. This is done by mechanically pressing only selected regions of the polyacrylate adhesive. Then, upon immersion in the salt solution, only the pressed regions are activated and promote NP growth therein, as illustrated by the examples of a fingerprint in Figure $5 \mathrm{c}$ and photolithographically defined patterns in Figure 5d. Third, when the tapes are decorated by Ag NPs, these particles confer their bacteriostatic and bacteriocidal effects ${ }^{15,16}$ on the polymeric surfaces, as illustrated in Figure 5b. Fourth, deposition of the metallic NPs increases the tape's electrical conductivity, as illustrated in Figure 5e $\left(4.81 \times 10^{-5} \mathrm{~S} / \mathrm{m}\right.$ for Au NP-decorated tape vs $1.15 \times 10^{-6} \mathrm{~S} / \mathrm{m}$ for the native tape). Importantly, all of these properties are in addition to the most practically important property, namely, the tape's remaining adhesive (cf. unchanged mechanical properties in Figure 3 and Figure S11).

In summary, we have described perhaps the most straightforward way of depositing metallic NPs onto polymer adhesives: in our mechanochemical approach, the target surface is activated by simple pulling of an adhesive tape. We have also demonstrated the combination of mechanoactivation and soft lithography - to the best of our knowledge, this is the first example of mechanochemical processes being micropatterned over target polymeric surfaces. Since mechanoradicals form on various types of polymers, ${ }^{4 a}$ our methods can be extended to other materials and systems. Some applications we envision as practically relevant are tapes covered with $\mathrm{Cu}$ NPs (for
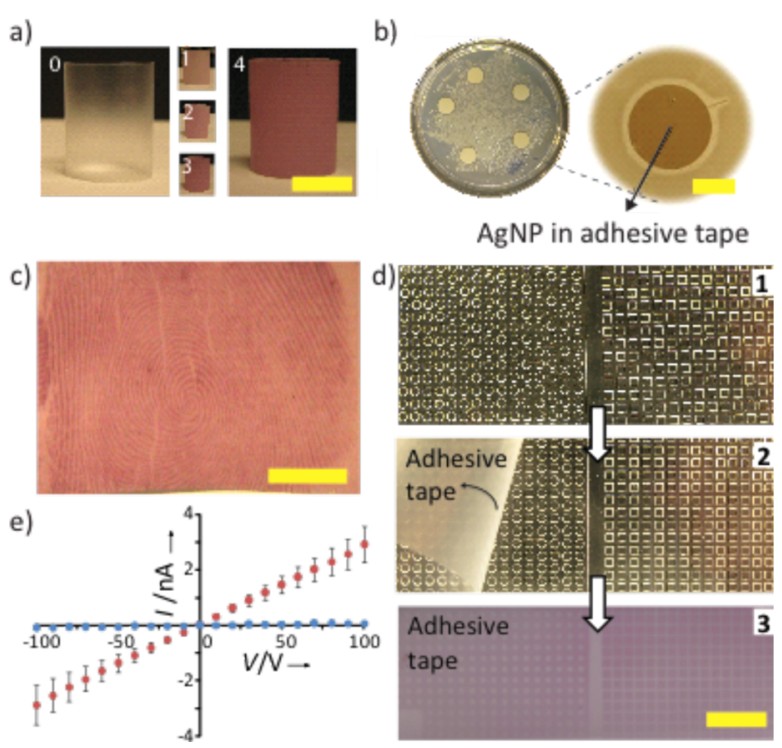

Figure 5. (a) Concentration of adsorbed NPs increases with immersion time (here $0-4$ days), as evidenced by the hue becoming deeper. Shown here are tapes harboring Au NPs. Scale bar $=1 \mathrm{~cm}$. (b) Tapes covered with Ag NPs show bacteriostatic properties, as evidenced by the clear (i.e., bacteria free) $1.6 \pm 0.4 \mathrm{~mm}$ thick zones of inhibition around circular tape pieces (see section 3 in the Supporting Information for details of this experiment). Scale bar $=0.5$ $\mathrm{cm}$. (c) NP growth is patterned by mechanically activating only select tape regions, here only at the locations where a finger was placed on the tape. The resulting deposition follows the fingerprint. Scale bar $=1$ $\mathrm{cm}$. (d) Regular patterns of NP deposition can also be obtained by pulling the tape off of polydimethylsiloxane (PDMS) masters and then immersing it in a salt solution. The PDMS master on the left has depressed "wells" on its surface-in these regions, the tape is not in contact with the PDMS and is not mechanoactivated for subsequent NP deposition. The PDMS master on the right has protruding posts that come into contact with the tape; again, only these regions are activated for NP deposition. Scale bar $=1 \mathrm{~cm}$. (e) NP-covered tapes become conductive. In the $I-V$ plot shown, blue symbols are for the original tape immersed in water for $24 \mathrm{~h}$ and red markers are for tape immersed in $2 \mathrm{mg} / \mathrm{mL} \mathrm{HAuCl}$ solution for $24 \mathrm{~h}$ (after immersion, both tapes were thoroughly dried overnight). The conductivity of the NP-decorated tape is $\sim 40$ times higher than of the "native" tape. Error bars are based on four independent measurements.

antifungal protection in moist areas) and bacteriostatic Ag NPbased films (e.g., Saran wrap) for antibacterial food packaging.

\section{ASSOCIATED CONTENT}

\section{(S) Supporting Information}

Further experimental details, instrumentation, and supporting figures. This material is available free of charge via the Internet at http://pubs.acs.org.

\section{AUTHOR INFORMATION}

\section{Corresponding Author}

*grzybor@northwestern.edu

\section{Notes}

The authors declare no competing financial interest.

\section{ACKNOWLEDGMENTS}

This work was supported by the Non-Equilibrium Energy Research Center (NERC), an Energy Frontier Research Center funded by the U.S. Department of Energy, Office of Science, Office of Basic Energy Sciences under Award DE-SC0000989. 
We thank Mustafa Guler (TEM imaging), Enver Kahveci (XPS and XRD measurements), and Dr. Bartlomiej Kowalczyk (SEM imaging) for their help in the analyses.

\section{REFERENCES}

(1) (a) Hajipour, M. J.; Fromm, K. M.; Ashkarran, A. A.; de Aberasturi, D. J.; de Larramendi, I. R.; Rojo, T.; Serpooshan, V.; Parak, W. J.; Mahmoudi, M. Trends Biotechnol. 2012, 30, 499. (b) Kon, K.; Rai, M. J. Clin. Pathol. 2013, 2, 160.

(2) Prabhu, S.; Poulose, E. K. Int. Nano Lett. 2012, 2, 32.

(3) (a) Schiffrin, D. J. MRS Bull. 2001, 26, 1015. (b) Lee, Y.; Choi, J.R; Lee, K. J.; Stott, N. E.; Kim, D. Nanotechnology 2008, 19, No. 415604.

(4) (a) Baytekin, H. T.; Baytekin, B.; Grzybowski, B. A. Angew. Chem., Int. Ed. 2012, 51, 3596. (b) Baytekin, H. T.; Patashinski, A. Z.; Branicki, M.; Baytekin, B.; Soh, S.; Grzybowski, B. A. Science 2011, 333, 308. (c) Baytekin, B.; Baytekin, H. T.; Grzybowski, B. A. J. Am. Chem. Soc. 2012, 134, 7223. (d) Baytekin, H. T.; Baytekin, B.; Hermans, T. M.; Kowalczyk, B.; Grzybowski, B. A. Science 2013, 341, 1368. (e) Baytekin, H. T.; Baytekin, B.; Grzybowski, B. A. Angew. Chem., Int. Ed. 2014, 53, 6946.

(5) Porthun, S.; Abelmann, L.; Lodder, C. J. Magn. Magn. Mater. 1998, 182, 238.

(6) (a) Marambio-Jones, C.; Hoek, E. M. V. J. Nanopart. Res. 2010, 12, 1531. (b) Rai, M.; Yadav, A.; Gade, A. Biotechnol. Adv. 2009, 27, 76.

(7) (a) Wei, Y.; Chen, S.; Kowalczyk, B.; Huda, S.; Gray, T. P.; Grzybowski, B. A. J. Phys. Chem. C 2010, 114, 15612. (b) Cioffi, N.; Torsi, L.; Ditaranto, N.; Tantillo, G.; Ghibelli, L.; Sabbatini, L.; BleveZacheo, T.; D’Alessio, M.; Zambonin, P. G.; Traversa, E. Chem. Mater. 2005, 17, 5255.

(8) Novoselov, K. S.; Geim, A. K.; Morozov, S. V.; Jiang, D.; Zhang, Y.; Dubonos, S. V.; Grigorieva, I. V.; Firsov, A. A. Science 2004, 306, 666.

(9) (a) Karasev, V. V.; Krotova, N. A.; Deryagin, B. W. Dokl. Akad. Nauk SSSR 1953, 88, 777. (b) Camara, C. G.; Escobar, J. V.; Hird, J. R.; Putterman, S. J. Nature 2008, 455, 1089. (c) Harvey, N. E. Science 1939, 89, 46. (d) Walton, A. J. Adv. Phys. 1977, 26, 887. (e) Zhenyi, M.; Fan, J.; Dickinson, J. T. J. Adhes. 1988, 25, 63. (f) Wu, F.-H.; Shen, S.-C.; Lee, L.-Y.; Lee, S.-H.; Chan, M. T.; Lin, C.-S. Plant Methods 2009, 5, 16. (g) Liang, J.; Ma, Y.; Wang, F.; Yang, W. Chem. Mater. 2010, 22, 4254.

(10) (a) Pittenger, B.; Erina, N.; Su, C. Quantitative Mechanical Property Mapping at the Nanoscale with PeakForce QNM; Bruker Application Note, 2010. (b) Rosa-Zeiser, A.; Weilandt, E.; Hild, S.; Marti, O. Meas. Sci. Technol. 1997, 8, 1333. (c) Sahin, O.; Magonov, S.; Chanmin, S.; Quate, C. F.; Solgaard, O. Nat. Nanotechnol. 2007, 2, 507. (d) Adamcik, J.; Berquand, A.; Mezzenga, R. Appl. Phys. Lett. 2011, 98, No. 193701.

(11) (a) Lacks, D. J.; Sankaran, R. M. J. Phys. D: Appl. Phys. 2011, 44, No. 453001. (b) Sakaguchi, M.; Shimad, S.; Kashiwabara, H. Macromolecules 1990, 23, 5038. (c) Wang, D.; Klaassen, A. A. K.; Janssen, G. E.; de Boer, E. Polymer 1995, 36, 4193. (d) Beyer, M. K.; Clausen-Schaumann, H. Chem. Rev. 2005, 105, 2911. (e) Potter, W. D.; Scott, G. Eur. Polym. J. 1971, 7, 489. (f) Porter, R. S.; Casale, A. Polym. Eng. Sci. 1985, 25, 129.

(12) McGilvray, K. L.; Decan, M. R.; Wang, D.; Scaiano, J. C. J. Am. Chem. Soc. 2006, 128, 15980.

(13) (a) Sarma, T. K.; Chowdhury, D.; Paul, A.; Chattopadhyay, A. Chem. Commun. 2002, 1048. (b) Panda, B. R.; Chattopadhyay, A. J. Nanosci. Nanotechnol. 2007, 7, 1911.

(14) (a) Ayrey, G.; Moore, C. G.; Watson, W. F. J. Polym. Sci. 1956, 19, 1. (b) Baranwal, K. J. Appl. Polym. Sci. 1968, 12, 1459. (c) Chandra, S.; Roy-Chowdury, P.; Biswas, A. B. J. Appl. Polym. Sci. 1964, 8, 2653. (d) Chandra, S.; Roy-Chowdury, P.; Biswas, A. B. J. Appl. Polym. Sci. 1966, 10, 1089. (e) Damm, C.; Peukert, W. Langmuir 2009, 25, 2264.

(15) Huda, S.; Smoukov, S. K.; Nakanishi, H.; Kowalczyk, B.; Bishop, K.; Grzybowski, B. A. ACS Appl. Mater. Interfaces 2010, 2, 1206.
(16) Lee, D.; Cohen, R. E.; Rubner, M. F. Langmuir 2005, 21, 9651. 